\title{
Neuro-fuzzy Modelling of a Linear Fresnel-type Solar collector System as A Digital Twin
}

\author{
*William D. Chicaiza and Adolfo J. Sánchez and Antonio J. Gallego and Juan M. Escaño \\ Department of System Engineering and Automatic Control, University of Seville, \\ Camino de los Descubrimientos s/n, 41092, Seville, Spain. \\ wchicaiza@us.es; asanchezdelpozo@us.es; agallego2@us.es; jescano@us.es
}

\begin{abstract}
One of the main components of a Digital Twin is the modeling of the virtual entity, being this a high-fidelity digital model of the physical entity that represents the modeling of geometry, modeling of physical properties, modeling of behavior, and modeling of rules in the virtual world.

This paper presents a model, based on an Adaptive Neuro-Fuzzy Inference System, of a Fresnel linear solar collector system as a Digital Twin, located on the roof of the School of Engineering of the University of Seville, which is a part of an absorption cooling plant.
\end{abstract}

A distributed parameter model of the system has been used to generate artificial data. Real operating data were used to validate the model.

Keywords: Digital Twin, Neuro-Fuzzy modeling, ANFIS.

\section{Introduction}

Digital Twin (DT) is one of the most promising technologies applied to smart manufacturing and industry 4.0. Several DT applications have been realized in product design, production and, prognostics and health management (PHM) thanks to the increasing technological development, information storage, and transmission speeds. The concept DT appeared in 2002 at the University of Michigan, promoting the premise that each system has a physical entity and a virtual entity. Term Digital Twin was included in Virtually Perfect for the conceptual model currently in use [11, 12]. The first general standard architecture that appears is modeling in three dimensions [11]. Similarly, [26] proposes a 5-layer DT modeling architecture, being this an extension of the three-dimensional architecture. There is also an eight-dimension architecture that describes the behavior and context of the DT [21].

Several are the works developed regarding DTs, in [12] argues that DT is the digital representation of a physical object by developing a DT of a bending test bench. Likewise, A step-by-step process of building a DT model is presented in [17] using a commercial drilling rig. Also, DT modeling of a 3D printing machine was performed in [7]. Industrial companies such as General Electric and Siemens have seen the great potential of DT [16, 27].

Deep learning shows the ability to learn and model large-scale data sets, unlike traditional learning methods [18]. The performance of a physical entity can be represented through artificial intelligence (AI) techniques such as fuzzy logic (FL), Artificial neural networks (ANN), neuro-fuzzy algorithms, or optimization techniques such as genetic algorithms (GA), particle swarm optimization (PSO), in order to solve many problems in different areas.

The advantages of fuzzy logic and artificial neural networks were concatenating in an AI technique called Neuro-Fuzzy. The most popular is the adaptive neurofuzzy inference system (ANFIS) proposed by Jang [14], which combines the learning capacity and relational structure of artificial neural networks with the decision-making mechanism of fuzzy logic [15, 9, 23]. Several papers use ANFIS [8] presents a neuro-fuzzy model of the temperature of an industrial autoclave to apply nonlinear predictive control. Used to predict the cone index values of arable soils [1], to control the bag process by implementing an ANFIS controller [3]. Similarly, ANFIS is used to forecast monthly water use $[28,4]$. In the energy context in [29], they investigated the interrelationship between energy consumption and economic growth in China. Successful applications of the neuro-fuzzy system for modeling complex nonlinear hydro-resource systems have been reported [2]. 
This work uses ANFIS to model an energy system, specifically the Linear Fresnel Collector (LFC) system of the ETSI of the University of Seville, which is part of the solar cooling plant installed. Since the dynamics of this kind of systems is highly nonlinear, a complex model is needed to describe this dynamics [6]. In order to develop optimal control and optimization strategies which use the prediction of the solar field temperature, a fast model is indispensable. The use of the nonlinear distributed parameter model to do this implies a high computational burden and this is a great hindrance if the problem has to be solved within a sampling time of several seconds [5]. Faster models are very important to be obtained. Thus the main objective of this paper is to obtain a neuro-fuzzy model that describes the performance of the real outlet temperature from the solar field, considering the variables that affect this process (solar radiation, water flow, ambient temperature, fluid temperature, and the local hour that will determine the position of the sun respect to the solar field).

The paper is organized as follows: Section 2 briefly describes the Fresnel solar field and its mathematical model. Section 3 describes the LFC ANFIS modeling. Section 4 shows the training and evaluation results. Finally, some concluding remarks are given in section 5 .

\section{Modeling of the Fresnel solar field}

This section presents a brief description of the Fresnel solar field and the mathematical model that will be used for the simulation of the plant and the generation of data to train the ANFIS models.

\subsection{Description of the Fresnel solar field}

The Escuela Técnica Superior de Ingeniería (ETSI) of Seville has a solar cooling plant (SCP). The SCP consists of three main subsystems: (1)Fresnel-type solar field, see Figure 1, (2) Absorption machine chiller and (3) a PCM storage tank. This work focuses on the LFC subsystem. The Fresnel solar field is installed on the roof of the building with an East-West orientation (Latitude $=37.4108972^{\circ}$, Longitude $=-6.0006621^{\circ}$ ) .

The solar field heats up the pressurized water coming from the chiller up to the required operation temperature $\left(145-165^{\circ} \mathrm{C}\right)$. The solar field consists of 11 rows of mirrors which focus the direct solar radiation on a metal tube of $64 \mathrm{~m}$ long whereby the water is circulating. A more complete description can be found in [19].

The modeling of the Fresnel field is developed using similar equations to the case of parabolic trough

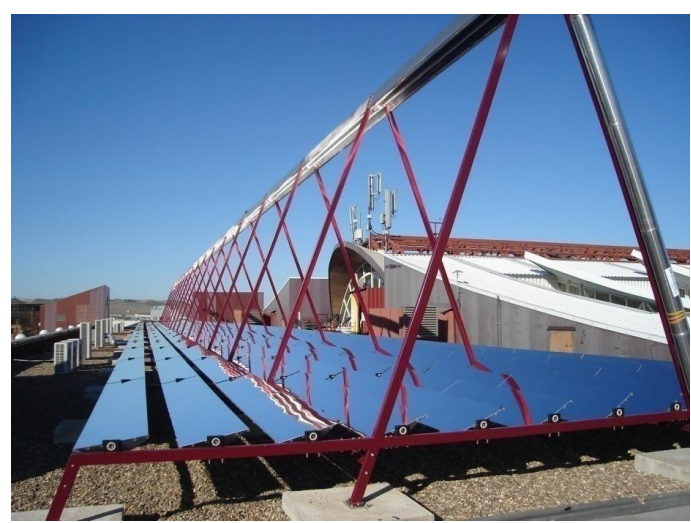

Figure 1: Fresnel solar collector.

fields modeling [24], but differ in the way of calculating the geometric efficiency, the shade factor and the thermodynamic properties of the fluid. The LFC system can be modeled using two different approaches: i) the concentrated parameter model used in control applications because its simplicity $[19,20,22]$ and ii) the distributed parameter model which takes into account the spatial distribution of the system providing a more precise description $[6,10,5]$. The distributed parameter model is used for simulation purposes.

\subsection{Distributed parameter model}

The distributed parameter model consists of a pair of differential equations in partial derivatives describing the energy balance [10] as follows:

$$
\begin{array}{r}
\rho_{m} C_{m} A_{m} \frac{\partial T_{m}}{\partial t}=I K_{o p t} n_{o} G-H_{l}\left(T_{m}-T_{a}\right) \ldots \\
\ldots-l_{p} H_{t}\left(T_{m}-T_{f}\right) \\
\rho_{f} C_{f} A_{f} \frac{\partial T_{f}}{\partial t}+\rho_{f} C_{f} q \frac{\partial T_{f}}{\partial l}=l_{p} H_{t}\left(T_{m}-T_{f}\right)
\end{array}
$$

where $m$ subindex refers to metal and $f$ subindex refers to fluid. The description of the parameters is presented in Table 1.

The calculation of the optical efficiency $K_{\text {opt }}$ requires knowledge of multiple factors such as the reflectivity of the mirror, the absorptance of the metal tube, the shape factor. The heat transmission coefficient, density and specific heat coefficient are obtained as polynomial functions of the segment temperature and the water flow by using thermodynamic data of the heat transfer fluid (pressurized water), which can be found in [6]. The geometric efficiency $n_{o}$ is obtained using complex trigonometric formulas, considering the effect of the cosine of the incidence angle of the solar 


\begin{tabular}{|c|c|c|}
\hline Symbol & Description & Units \\
\hline$t$ & Time & $s$ \\
$l$ & Space & $m$ \\
$\rho$ & Density & $\mathrm{kg} \mathrm{m}^{3}$ \\
$C$ & Specific heat capacity & $\mathrm{JK}^{-1} \mathrm{~kg}^{-1}$ \\
$A$ & Cross sectional area & $\mathrm{m}^{2}$ \\
$T(l, t)$ & Temperature & ${ }^{\circ} \mathrm{K}^{\circ} \mathrm{C}$ \\
$q(t)$ & Water flow rate & $\mathrm{m}^{3} \mathrm{~s}^{-1}$ \\
$I(t)$ & Direct solar radiation & $\mathrm{Wm}^{2}$ \\
$n_{o}$ & Geometric efficiency & Unitless \\
$K_{o p t}$ & Optical efficiency & Unitless \\
$G$ & Collector aperture & $\mathrm{m}$ \\
$T_{a}(t)$ & Ambient temperature & ${ }^{\circ} \mathrm{K}^{\circ} \mathrm{C}$ \\
$H_{l}$ & Global coefficient & $\mathrm{Wm}^{-2}{ }^{\circ} \mathrm{C}^{-1}$ \\
& of thermal loss & \\
$H_{t}$ & Coefficient of heat & $\mathrm{Wm}^{-2}{ }^{\circ} \mathrm{C}^{-1}$ \\
& transmission metal-fluid & \\
$l_{p}$ & Length of pipe line & $\mathrm{m}$ \\
$S$ & Total reflective surface & $\mathrm{m}^{2}$ \\
\hline
\end{tabular}

Table 1: Description of the parameters.

beam and the shade factor [19]. The distributed parameter model is solved with an integration time of $0.25 \mathrm{~s}$, dividing the tube into 64 segments of $1 \mathrm{~m}$ each. The distributed parameter model is used as a simulation model to generate artificial data in order to be used to train the Neuro-fuzzy model.

\section{LFC ANFIS modelling}

ANFIS integrates two methods of Soft-Computing: artificial neural networks (ANN) and a fuzzy inference system (FIS) [14]. Providing a method applicable to fuzzy modeling, learning from a data set in order to calculate the parameters of the membership functions (MF) that best allow the associated FIS to follow the input-output data $[23,13]$. Fuzzy logic (FL) can model arbitrarily complex non-linear functions. Non-linearity and complexity are handled by rules, MF and the inference process, improving performance with simpler implementation. The fuzzy Takagi-Sugeno model can be formulated as an ANFIS [25], except that the links indicate the direction of signal flow and no associated weights. The ANFIS architecture consists of five layers: fuzzification, product, normalization, defuzzification and output. The first and fourth layers contain adaptive nodes (square nodes) that represent the parameter sets that are adjustable, while the remaining layers contain fixed nodes (circular nodes) that represent the parameter sets that are fixed in the system [14].

ANFIS requests a training data set, $\left(x_{1}, x_{2}, \cdots, y\right)$ desired input-output pair that represents the system to model. Obtaining a dynamic model is not always easy, either because of the complexity, randomness, or lack of knowledge of the study system. An accurate model in many cases will consist of a high number of equations, but in some cases, it is not always adequate to solve uncertain systems.. The use of an ANFIS to model the qualitative aspects and reasoning processes of human knowledge, using fuzzy rules to describe the performance of the system, and avoiding precise quantitative analysis is an alternative.

ANFIS starts from previous knowledge, or the necessary information can be added to improve the model. In this work with an ANFIS, a set of rules describing the dynamics of the solar field output temperature has been obtained. When defining its structure we are faced with the drawback of setting the MFs, since a greater number of MFs determines greater knowledge. However it generates the use of numerous rules, this can be solved by applying clustering methods that seek to classify data into subsets.

\subsection{Input/Output data set selection}

The neuro-fuzzy model represents the performance of the real outlet temperature from the solar field, using the variables that influence this process: solar radiation, water flow, ambient temperature, fluid temperature, and the local hour that will determine the position of the sun. The solar field evolves independently in an open-loop from an arbitrarily given starting point to obtain data for subsequent ANFIS training.

The distributed parameter model has been used to simulate the solar plant and obtain artificial data that will be part of the training set. Simulations have been carried out, starting from the system in a permanent state. Initially, there has only been one input change in each variable, of the step type, while the rest of the variables remain constant in average values. Likewise, the levels of the amplitudes of the steps were divided into three regions (low, medium, high) and these have been given for the variables water flow, inlet fluid temperature and solar radiation. The values of the applied steps have thus served to better capture the dynamics of the system, in the formation of the ANFIS.

Real and artificial input-output data from the solar field has been used, to prepare two data sets: training and checking set. The training set is composed of real data from two different days in the months of June, July as shown in Figure 2(a) and artificial data obtained from the distributed parameter model, see Figure 2(b). While the checking data is composed of real data from two different days in the month of August as shown in Figure 3. The training set must contain all the representative characteristics of the system to be modeled. 
This set is used by ANFIS to find a spatial input-output relationship through repetitive analysis.
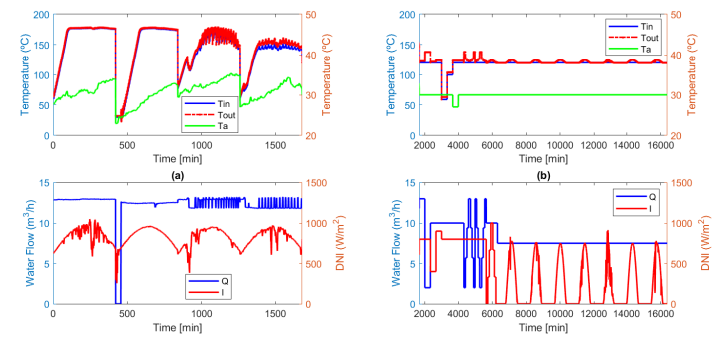

Figure 2: Training data set: (a) real data, (b) artificial data

The checking set allows to verify the generalization capacity of the obtained ANFIS model, should be different from the training set, so that the validation process is not trivial and avoids overfitting. Figures 2 and 3 shows training and checking data.
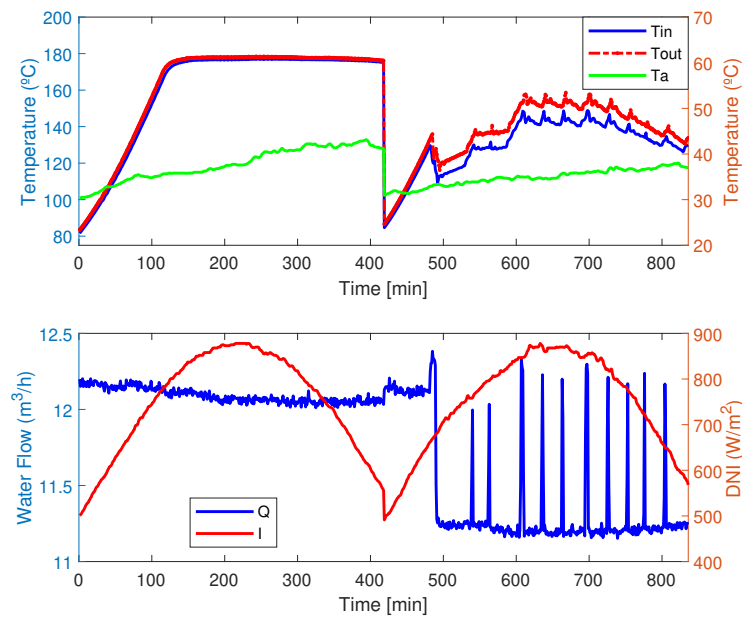

Figure 3: Checking data set.

\subsection{Data processing}

A process of normalization of the input-output data set is performed. The use of normalized values prevents the different nature and magnitude of the variables from affecting the neural learning process, also helps to reduce noise, inconsistency and leads to a better estimation of the modeling.

The original data has been normalized in the range [0 1] using Eq. 2.

$$
X_{\text {norm }}=\frac{x-x_{\min }}{x_{\max }-x_{\min }}
$$

Where $x$ is the data to be normalized, $\left(x_{\max }, x_{\min }\right)$ is the maximum and minimum of the original data respectively and $X_{\text {norm }}$ is the normalized data.

ANFIS has been provided with memory through a recursive structure, using past values of the inputs and outputs to capture the solar field dynamics. There are no standard methods to transform human knowledge or experience into a base of rules and data. Therefore, it becomes the art of adjusting the ANFIS parameters in order to minimize the error or maximize the performance index.

\section{Results}

This section presents the results of the ANFIS models. Simulations have been carried out with real data from different days of September where the real results will be compared with those obtained with the ANFIS models.

\subsection{ANFIS training results}

The training process starts with seven inputs with a hybrid configuration [14] and the subtractive clustering (SC) method with a influence range equal to 0.70 . A total of four Gaussian type MFs are obtained for each input. The number of epochs used during training was 200 and the evaluation criterion for estimating accuracy of the model is performed by comparing the RMSE at each epoch. However, as can be observed in the ANFIS modeling parameters, the influence range of clustering, and the number of MFs have been varied in order to obtain a more generalized learning of the training and checking sets. The ANFIS architecture is also modified when the $R M S E_{C h k}$ is considerable during training. The number of rules is changed since redundant rules lead to overfitting. Overfitting indicates that the ANFIS model obtained is fitted too closely to the training data set and the final performance of the model with respect to another data set will be deficient. The Figure 4 shows the overfitting effect considering a high number of epochs $\left(k=10 e^{4}\right)$, since the learning rate also depends on the number of given epochs.

If $k$ is small the convergence will be slow but if $k$ is large the convergence will be initially very fast and will oscillate around the optimum. However, for a very high $k$ the ANFIS model is fitted the training data and the $R M S E_{C h k}$ increases.

The obtained ANFIS architecture of the model is shown in Table 2 and Figure 5 shows the evolution of the training and checking RMSE index at each epoch. The RMSE checking curve has to be observed more to evaluate the accuracy of the model, since it indicates 


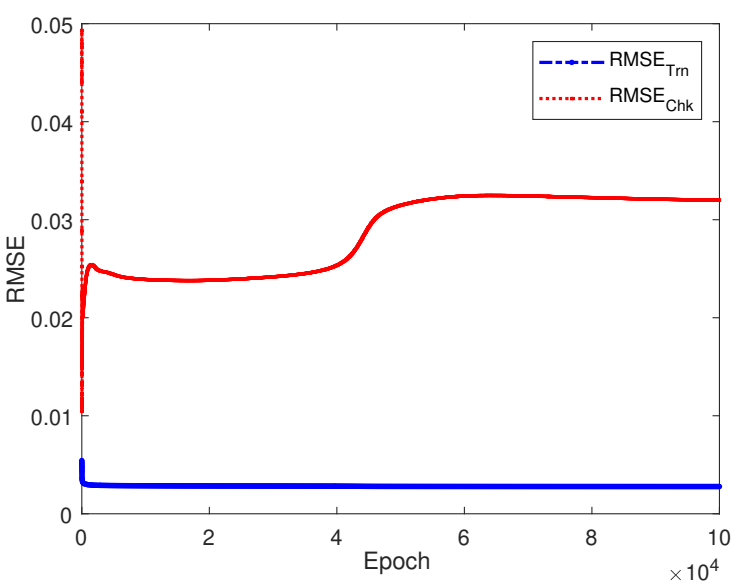

Figure 4: Overfitting effect on ANFIS modeling training.

if the ANFIS learning is general ${ }^{1}$. Therefore, a middle point is sought where the learning is general for both sets. For the rest of the paper this model will be named as ANFIS1.

\begin{tabular}{|c|c|}
\hline Description & RMSE min \\
\hline Number MFs: 3 Gaussiantype & Normalized data \\
Number rules: 3 & $R M S E_{T r n}=0.0028$ \\
Optimization method: hybrid & $R M S E_{C h k}=0.0105$ \\
Output MF type: linear & \\
Influence range: 0.72 & \\
Epoch number: 200 & \\
\hline
\end{tabular}

Table 2: ANFIS1 architecture parameters obtained with normalized data.

\subsection{ANFIS evaluation results}

The results of the evaluation of the model obtained are presented when using real data from days of operation. Two error indexes were used to compare the models, the RMSE and the MAPE, Eqs. 3 and 4.

$$
\begin{gathered}
R M S E=\sqrt{\frac{\sum_{t=1}^{n}\left(x_{t}-x_{t}^{\prime}\right)^{2}}{n}} \\
M A P E=\frac{\sum_{t=1}^{n}\left|\frac{x_{t}-x_{t}^{\prime}}{x_{t}}\right|}{n}
\end{gathered}
$$

We performed a simulation to evaluate the ANFIS1 model. Figure 6 shows that ANFIS1 model has a good

\footnotetext{
${ }^{1}$ If $R M S E_{T r n}$ down and $R M S E_{C h k}$ up, the ANFIS modeling is learning from one set but gets more error in the other, which indicates that learning is not general.
}

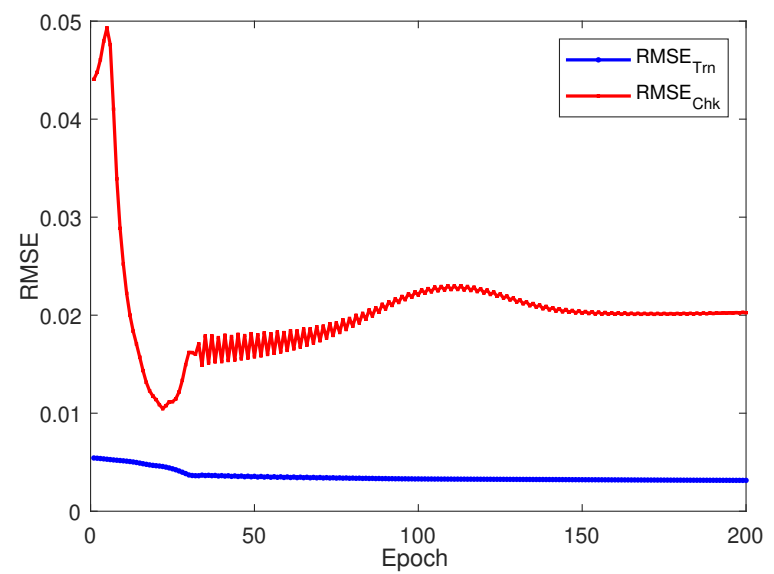

Figure 5: Training and checking RMSE curves for ANFIS1 modeling.

performance capturing the dynamics of the LFC field except in the last part of the day, where the error is higher. Figure 7 shows the results of the ANFIS1 model and real data from a different day. It can show that, although the dynamics are similar, the error in this simulation is very high.

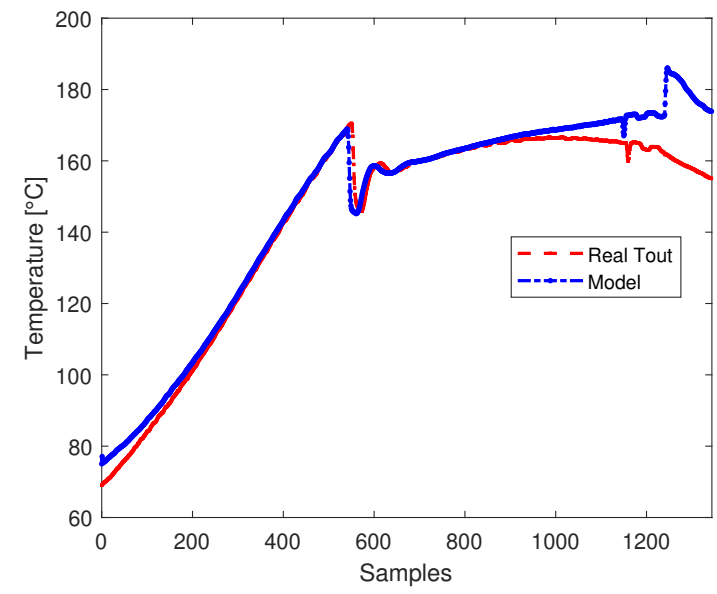

Figure 6: ANFIS1 model evaluation, model data vs real data. Day data-set 1 .

In order to obtain a model with a better overall performance, a second ANFIS (ANFIS2) has been trained with a different architecture and parameters which are shown in Table 3. The number of epochs has been increased and the range of clustering influence has been modified to 0.70 for this ANFIS model.

It can be checked, comparing tables 2 and 3, that the RMSE (Trn and Chk) of ANFIS2 are similar to those of ANFIS1. The RMSE curves of training and checking are shown in Figure 8. 


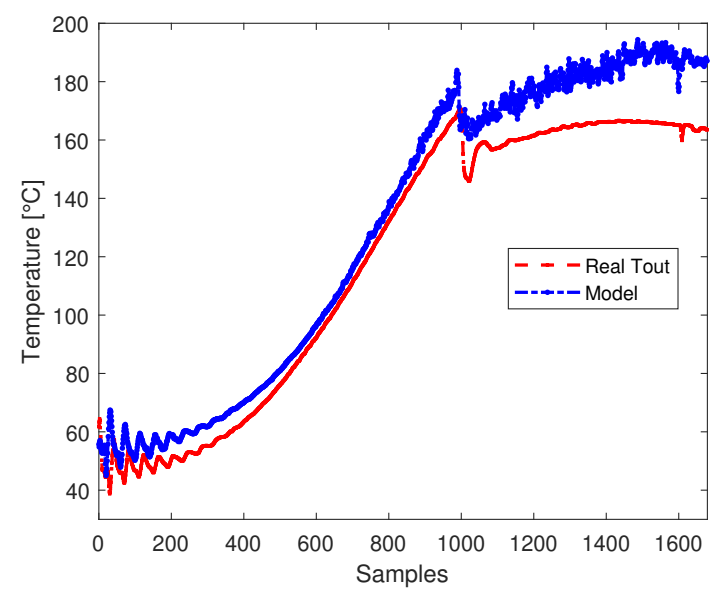

Figure 7: ANFIS1 model evaluation, model data vs real data. Day data-set 2.

\begin{tabular}{|c|c|}
\hline Description & RMSE min \\
\hline Number MFs: 4 Gaussiantype & Normalized data \\
Number rules: 4 & $R M S E_{T r n}=0.0026$ \\
Optimization method: hybrid & $R M S E_{C h k}=0.0106$ \\
Output MF type: linear & \\
Influence range: 0.70 & \\
Epoch number: 1000 & \\
\hline
\end{tabular}

Table 3: ANFIS2 architecture parameters.

Figure 9 show the evolution of ANFIS2 model vs the real data, for day data-set 1 . as in the previous case (ANFIS1), showing that ANFIS2 model also provides a good performance in capturing the LFC system dynamics but with a better performance along the day. ANFIS2 model has also been evaluated in another day of real data day data-set 2, shown in Figure 10, where it can be observed the good performance of the model evolution along the day.

In order to compare the results of both models (ANFIS1 and ANFIS2), a set of three simulations results are shown in Table 4 where the RMSE and MAPE indexes are shown for the different test days. It can be observed that ANFIS2 model presents lower error rates than ANFIS1, concluding that this model presents better results in the estimation of the outlet temperature from the solar field.

ANFIS2 captures the actual output temperature dynamics of the solar field in a better way, with twice as many epochs compared to the previous model. However, the benefit obtained is appreciable with the advantage of not having a very high number of epochs. The optimum number of epochs would be in this range. Regarding the influence range, the optimum range

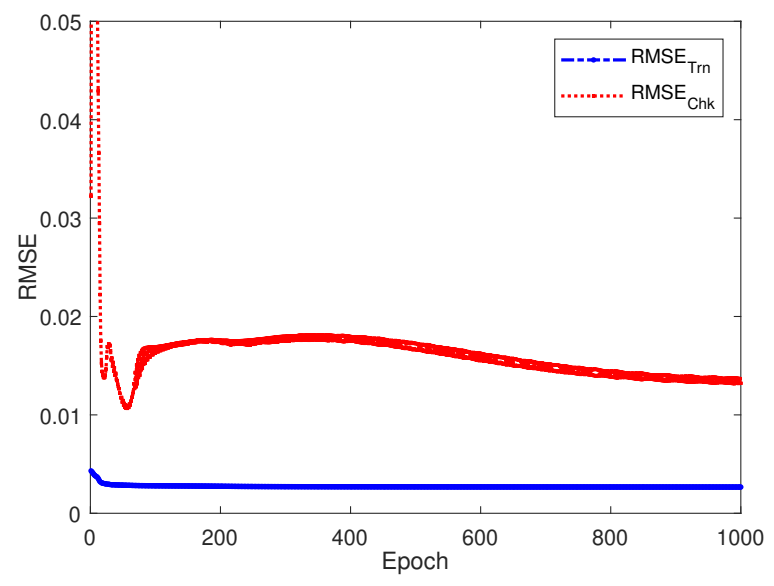

Figure 8: Training and checking RMSE curves for best ANFIS modeling with normalized data.

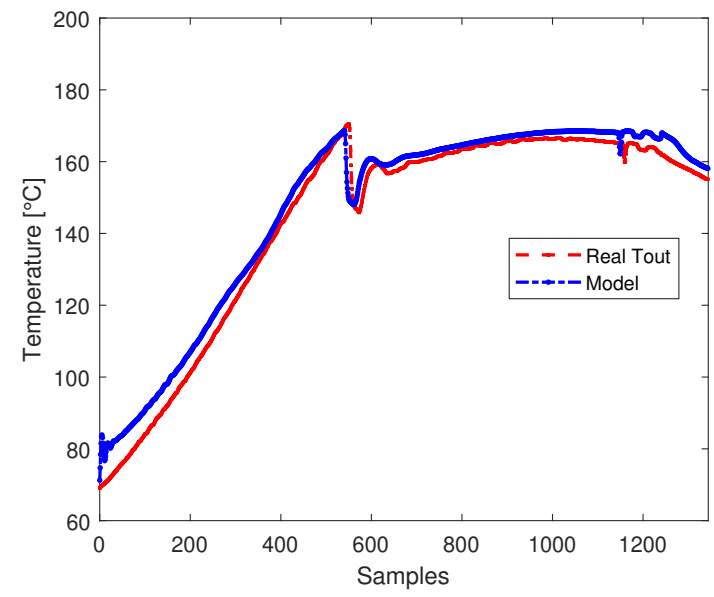

Figure 9: ANFIS2 model evaluation, model data vs real data. Day data-set 1 .

should be in the interval $[0.7-0.72]$.

Finally, a simulation of the distributed parameter model and the ANFIS2 neuro-fuzzy model has been performed to compare the computational load of each of them. The execution time of the distributed parameter model was $35.03[\mathrm{~ms}]$ while that of the ANFIS2 system was 634.98[us]. In conclusion, the computational burden of ANFIS2 concerning the distributed parameter model is 55 times faster, which makes it suitable for real-time control applications where the dynamic model of the plant is required. Also, the number of learning parameters of ANFIS is much lower concerning the distributed parameter model. 


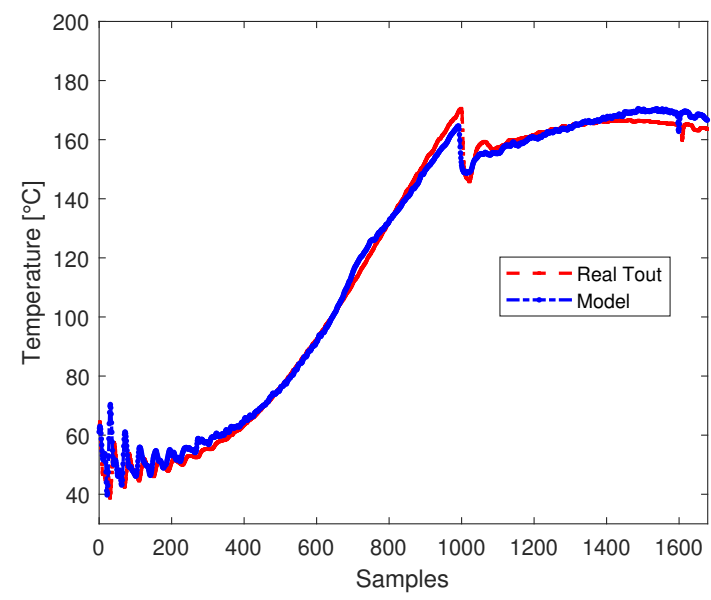

Figure 10: ANFIS2 model evaluation, model data vs real data. Day data-set 2 .

\begin{tabular}{|c|c|c|}
\hline $\begin{array}{c}\text { Error } \\
\text { indexes }\end{array}$ & $\begin{array}{c}\text { ANFIS1 } \\
\text { model }\end{array}$ & $\begin{array}{c}\text { ANFIS2 } \\
\text { model }\end{array}$ \\
\hline Day & \multicolumn{2}{|c|}{ Test 1 } \\
\hline$R M S E$ & 6.9890 & 4.42 \\
$M A P E$ & $2.69 \%$ & $2.27 \%$ \\
\hline Day & \multicolumn{2}{|c|}{ Test 2 } \\
\hline$R M S E$ & 12.9572 & 3.6726 \\
$M A P E$ & $8.54 \%$ & $2.58 \%$ \\
\hline Day & \multicolumn{3}{|c|}{ Test 3 } \\
\hline$R M S E$ & 8.58 & 7.5141 \\
$M A P E$ & $5.69 \%$ & $4.51 \%$ \\
\hline
\end{tabular}

Table 4: RMSE and MAPE indexes obtained for ANFIS1 and ANFIS2.

\section{Conclusion}

In this work ANFIS has been applied to capture the dynamics of real outlet temperature from a Fresnel-type solar field, obtaining satisfactorily a series of models which differ in the number of rules.Specifically, the range of influence of clustering and the number of epochs have been varied to obtain a better model with a lower error coefficient at the instant of evaluation.

The use of output feedback allows the capture of the dynamics of the process to be modelled. The result is a recurrent neural network, whose convergence is more complex. The ANFIS model obtained in this work is auto-regressive of second order.

Modifying various parameters, several ANFIS models have been obtained. One of them has been chosen, with an acceptable validation error, to follow the dynamic evolution of the process. This paper lays the foundations for creating a framework with which to de- sign ANFIS-based digital twins for plants of this type. In future works, the error must be improved by learning with continuous operating data.

\section{Acknowledgement}

The authors want to thank the European Commission for funding this work under project DENiM. This project has received funding from the European Union's Horizon 2020 research and innovation programme under grant agreement No 958339.

\section{References}

[1] M. Abbaspour-Gilandeh, Y. AbbaspourGilandeh, Modelling soil compaction of agricultural soils using fuzzy logic approach and adaptive neuro-fuzzy inference system (ANFIS) approaches, Modeling Earth Systems and Environment 5 (1) (2019) 13-20.

[2] M. Aqil, I. Kita, A. Yano, S. Nishiyama, A comparative study of artificial neural networks and neuro-fuzzy in continuous modeling of the daily and hourly behaviour of runoff, Journal of Hydrology 337 (1) (2007) 22 - 34.

[3] G. S. Atsalakis, K. P. Valavanis, Forecasting stock market short-term trends using a neurofuzzy based methodology, Expert Systems with Applications 36 (7) (2009) 10696-10707.

[4] A. Azadeh, M. Saberi, S. Asadzadeh, An adaptive network based fuzzy inference system-auto regression-analysis of variance algorithm for improvement of oil consumption estimation and policy making: The cases of canada, united kingdom, and south korea, Applied Mathematical Modelling 35 (2) (2011) 581 - 593.

[5] E. F. Camacho, A. J. Gallego, J. M. Escaño, A. J. Sánchez, Hybrid nonlinear MPC of a solar cooling plant, Energies 12 (14).

[6] E. F. Camacho, A. J. Gallego, A. J. Sanchez, M. Berenguel, Incremental state-space model predictive control of a fresnel solar collector field, Energies 12 (1) (2019) 1-23.

[7] T. DebRoy, W. Zhang, J. Turner, S. S. Babu, Building digital twins of 3D printing machines, Scripta Materialia 135 (2017) 119-124.

[8] J. Escaño, C. Bordons, M. R. Garcia, C. Vilas, A. Alonso, Control predictivo basado en modelo neuroborroso de un autoclave industrial, 2007, xXVIII Jornadas de Automática. 
[9] N. G. Fragiadakis, V. D. Tsoukalas, V. J. Papazoglou, An adaptive neuro-fuzzy inference system (anfis) model for assessing occupational risk in the shipbuilding industry, Safety Science 63 (2014) 226-235.

[10] A. J. Gallego, A. J. Sánchez, M. Berenguel, E. F. Camacho, Adaptive ukf-based model predictive control of a fresnel collector field, Journal of Process Control 85 (2020) 76 - 90.

[11] M. Grieves, J. Vickers, Digital twin: Mitigating unpredictable, undesirable emergent behavior in complex systems, in: F.-J. Kahlen, S. Flumerfelt, A. Alves (Eds.), Transdisciplinary Perspectives on Complex Systems: New Findings and Approaches, Springer International Publishing, Cham, 2017, pp. 85-113.

[12] S. Haag, R. Anderl, Digital twin - Proof of concept, Manufacturing Letters 15 (2018) 64-66.

[13] M. Imran, S. A. Alsuhaibani, Chapter 7 - a neurofuzzy inference model for diabetic retinopathy classification, in: D. J. Hemanth, D. Gupta, V. Emilia Balas (Eds.), Intelligent Data Analysis for Biomedical Applications, Intelligent DataCentric Systems, Academic Press, 2019, pp. 147 -172 .

[14] J. . R. Jang, Anfis: adaptive-network-based fuzzy inference system, IEEE Transactions on Systems, Man, and Cybernetics 23 (3) (1993) 665-685.

[15] D. Karaboga, E. Kaya, Adaptive network based fuzzy inference system (ANFIS) training approaches: a comprehensive survey, Artificial Intelligence Review 52 (4) (2019) 2263-2293.

[16] A. M. Lund, K. Mochel, J.-W. Lin, R. Onetto, J. Srinivasan, P. Gregg, J. E. Bergman, K. D. Hartling, A. Ahmed, S. Chotai, Digital wind farm system, US Patent US20160333855A1.

[17] A. Moreno, G. Velez, A. Ardanza, I. Barandiaran, Á. R. de Infante, R. Chopitea, Virtualisation process of a sheet metal punching machine within the Industry 4.0 vision, International Journal on Interactive Design and Manufacturing 11 (2) (2017) 365-373.

[18] B. Rezaeianjouybari, Y. Shang, Deep learning for prognostics and health management: State of the art, challenges, and opportunities, Measurement: Journal of the International Measurement Confederation 163 (2020) 107929.
[19] M. Robledo, J. M. Escaño, A. Núñez, C. Bordons, E. F. Camacho, Development and experimental validation of a dynamic model for a fresnel solar collector, Vol. 44, 2011, pp. 483 - 488, 18th IFAC World Congress.

[20] M. Spoladore, E. F. Camacho, M. E. Valcher, Distributed parameters dynamic model of a solar fresnel collector field, in: IFAC Proceedings Volumes (IFAC-PapersOnline), Vol. 44, IFAC Secretariat, 2011, pp. 14784-14789.

[21] R. Stark, C. Fresemann, K. Lindow, Development and operation of Digital Twins for technical systems and services, CIRP Annals 68 (1) (2019) 129-132.

[22] P. Sutcliffe, R. M. Rennie, E. J. Jumper, Development and experimental validation of a dynamic model for wind-tunnel heat exchangers, 53rd AIAA Aerospace Sciences Meeting.

[23] I. Svalina, V. Galzina, R. Lujić, G. Šimunović, An adaptive network-based fuzzy inference system (ANFIS) for the forecasting: The case of close price indices, Expert Systems with Applications 40 (15) (2013) 6055-6063.

[24] A. Sánchez, A. Gallego, J. Escaño, E. Camacho, Thermal balance of large scale parabolic trough plants: A case study, Solar Energy 190 (2019) 69 -81 .

[25] T. Takagi, M. Sugeno, Fuzzy identification of systems and its applications to modeling and control, IEEE Transactions on Systems, Man, and Cybernetics SMC-15 (1) (1985) 116-132.

[26] F. Tao, M. Zhang, Y. Liu, A. Y. Nee, Digital twin driven prognostics and health management for complex equipment, CIRP Annals 67 (1) (2018) 169-172.

[27] L. Wang, A. M. Canedo, Human programming interfaces for machine-human interfaces, US Patent US20170108834A1.

[28] M. A. Yurdusev, M. Firat, Adaptive neuro fuzzy inference system approach for municipal water consumption modeling: An application to Izmir, Turkey, Journal of Hydrology 365 (3-4) (2009) 225-234.

[29] X. Zhang, Y. Mao, The relationship between energy consumption and economic growth in china based on anfis, in: 2009 International Workshop on Intelligent Systems and Applications, 2009, pp. $1-4$. 